hep-th/0305175

\title{
Dimensional reductions of M-theory S-branes to string theory $\mathrm{S}$-branes
}

\author{
Shibaji Roy ${ }^{1}$ \\ Saha Institute of Nuclear Physics \\ 1/AF Bidhannagar, Calcutta-700 064, India
}

\begin{abstract}
We study both the direct and the double dimensional reduction of space-like branes of Mtheory and point out some peculiarities in the process unlike their time-like counterpart. In particular, we show how starting from SM2 and SM5-brane solutions we can obtain SD2 and SNS5-brane as well as SNS1 and SD4-brane solutions of string theory by direct and double dimensional reductions respectively. In the former case we need to use delocalized SM-brane solutions, whereas in the latter case we need to use anisotropic SM-brane solutions in the directions which are compactified.
\end{abstract}

\footnotetext{
${ }^{1}$ E-Mail: roy@theory.saha.ernet.in
} 


\section{Introduction}

Space-like branes [1] (or Sp-branes) are a class of time-dependent solutions of string/M theory (also of some field theories) which are subject of much interests in recent times. $\mathrm{S} p$-branes are topological defects localized in $(p+1)$ dimensional space-like surfaces and exist at a moment in time. They can be understood to appear as a time-like tachyonic kink solution of unstable $\mathrm{D}(p+1)$-brane in string theory [2] and is believed to shed light on dS/CFT correspondence $[3,4]^{2}$. As for other time dependent solutions [6] S-branes ${ }^{3}$ are also interesting from the cosmological point of view [8]. Various aspects of S-branes in $\mathrm{M} /$ string theory have been explored in [9, 10, 11, 12.

Just like in static case M-theory has two kinds of S-branes, namely, SM2 and SM5branes and are characterized by two parameters. On the other hand, string theory has SNS1, SNS5 and SD -branes (as their time-like counterpart) characterized by four parameters. These solutions were obtained in [9] by solving the equations of motion of the corresponding supergravity actions. Since type IIA string theory can be obtained as $\mathrm{S}^{1}$ reduction of M-theory, the M-theory S-branes SM2 must reduce to SD2 of type IIA string theory under direct dimensional reduction [13] and to SNS1 under double dimensional reduction [14 as in the static case. Similarly, SM5-brane should reduce to SNS5-brane of type IIA string theory under direct dimensional reduction and SD4-brane under double dimensional reduction as is known for the static case. However, the reduction procedure can not be the same since the M-theory S-branes are two parameter solution, whereas the string theory solutions are four parameter solutions [9]. The naive dimensional reduction can not produce new parameters in the reduced solutions.

The purpose of this paper is to show how starting from M-theory S-branes one can reproduce the various string theory S-branes under dimensional reductions since all Mtheory and string theory S-brane solutions are known explicitly. For the BPS timelike branes, the direct dimensional reduction is performed along a transverse space-like isometric direction, where isometry is produced by placing parallel branes periodically along the to be compactified transverse direction. However, for the space-like branes this procedure is not well-defined, so, instead we use a delocalized SM2 and SM5-brane solutions (as has been obtained in 9 with a modification) along the to be compactified transverse direction. We will show that the direct dimensional reduction of this solution correctly reproduces the known SD2 4 and SNS5-brane solution of type IIA string theory.

\footnotetext{
${ }^{2}$ In three dimensions the dS/CFT correspondence has been noted earlier in [5].

${ }^{3}$ See 7 for some earlier works.

${ }^{4}$ This case has also been studied in [15].
} 
The double dimensional reduction for the time-like branes is much easier to perform since here the compactification is done along one of the space-like directions of the brane which is already an isometric direction. For the space-like branes although the procedure is similar, but we will show that we must start from anisotropic (in the to be compactified longitudinal direction of the brane) SM-brane solutions in order to reproduce both the SNS1 and SD4 solutions of type IIA string theory under double dimensional reductions.

The organization of this paper is as follows. In section 2, we write the localized $\mathrm{S} p$-brane solutions of the gravity coupled to the dilaton and an $n$-form field strength in $d=p+n+2$ dimensions. In $d=11,10$ they represent various S-brane solutions of $\mathrm{M} /$ string theory in question. In section 3 , we discuss the direct dimensional reductions. The double dimensional reductions are discussed in section 4 . We conclude in section 5 .

\section{Localized S $p$-brane solutions}

In this section, we write the localized Sp-brane solutions obtained in 9] (by setting $k=q$ there) by solving the equations of motion resulting from $d$-dimensional gravity coupled to the dilaton and an $n$-form field strength. The solution in space-time dimensions $d=$ $p+n+2$ has the symmetry $\operatorname{ISO}(p+1) \times \operatorname{SO}(n, 1), \operatorname{ISO}(p+1) \times \operatorname{ISO}(n)$ or $\operatorname{ISO}(p+1) \times$ $\mathrm{SO}(n+1)$ depending on whether the internal spaces are hyperbolic, flat or spherical and takes the form,

$$
\begin{aligned}
d s_{d}^{2} & =-e^{2 A(t)} d t^{2}+e^{2 B(t)}\left(d x_{1}^{2}+\cdots+d x_{p+1}^{2}\right)+e^{2 C(t)} d \Sigma_{n, \sigma}^{2} \\
\phi & =\frac{a(d-2)}{n-1} B(t)+c_{1} t+c_{2} \\
F_{n} & =b \epsilon\left(\Sigma_{n, \sigma}\right)
\end{aligned}
$$

In the above $A, B$ and $C$ are functions of time satisfying a gauge condition

$$
-A+(p+1) B+n C=0
$$

In this gauge the equations of motion simplify and the three functions can be expressed in terms of two independent functions as,

$$
A=n g(t)-\frac{p+1}{n-1} f(t), \quad B=f(t), \quad C=g(t)-\frac{p+1}{n-1} f(t)
$$

Also in the above $d \Sigma_{n, \sigma}^{2}$ is the line element of the hyperbolic $(\sigma=-1)$, flat $(\sigma=0)$ and spherical $(\sigma=+1)$ space, $a$ is the dilaton coupling to the $n$-form field strength, $b$ is the 
field strength parameter and $\epsilon\left(\Sigma_{n, \sigma}\right)$ is the unit volume element of $\Sigma_{n, \sigma}$. By solving the equations of motion the functions $f(t)$ and $g(t)$ are obtained as,

$$
\begin{aligned}
& f(t)=\frac{2}{\chi} \ln \frac{\alpha}{\cosh \left[\frac{\chi \alpha}{2}\left(t-t_{0}\right)\right]}+\frac{1}{\chi} \ln \frac{(d-2) \chi}{(n-1) b^{2}}-\frac{a}{\chi}\left(c_{1} t+c_{2}\right) \\
& g(t)=\left\{\begin{array}{lll}
\frac{1}{(n-1)} \ln \frac{\beta}{\sinh [(n-1) \beta|t|]}, & \text { for } & \sigma=-1 \\
\pm \beta t, & \text { for } & \sigma=0 \\
\frac{1}{(n-1)} \ln \frac{\beta}{\cosh [(n-1) \beta t]}, & \text { for } & \sigma=+1
\end{array}\right.
\end{aligned}
$$

where $\alpha, \beta, c_{1}, c_{2}, t_{0}$ are integration constants. $\chi$ is defined as,

$$
\chi=2(p+1)+\frac{a^{2}(d-2)}{n-1}
$$

and the constants satisfy the following relation,

$$
\frac{p+1}{\chi} c_{1}^{2}+\frac{(d-2) \chi \alpha^{2}}{2(n-1)}-n(n-1) \beta^{2}=0
$$

This is the complete localized $\mathrm{S} p$-brane solution in $d$ space-time dimensions. For $d=11$, $a=c_{1}=c_{2}=0$, we get localized SM2-brane solution for $p=2$ and $n=7$ (also $\chi=6$ ) and SM5-brane solution for $p=5$ and $n=4(\chi=12$ in this case). On the other hand, for $d=10$ we get localized SD2 solution for $p=2, n=6, a=-1 / 2, \chi=32 / 5$ and SNS5 solution for $p=5, n=3, a=-1, \chi=16$. We also get SNS1 solution for $p=1, n=7$, $a=1, \chi=16 / 3$ and SD4 solution for $p=4, n=4, a=1 / 2, \chi=32 / 3$. Although we will not use the localized SM-brane solutions for dimensional reduction as they will not produce the correct localized string theory S-brane solutions, we will comment on them later for comparison. The localized string theory S-brane solutions as given in (2.1) (2.6) for various values of $p, n, a$ and $\chi$ will be compared after the dimensional reduction of appropriate M-theory S-branes. We comment that M-theory S-branes are characterized by the parameters $\alpha, \beta, t_{0}$ and $b$ with a relation between $\alpha$ and $\beta$ given by (2.6). But $\beta$ can be eliminated from the solution by appropriate scaling of $t$ and $x_{i}$ coordinates. So, the solution would depend on two parameters. Similarly, the string theory solution can be shown [11] to depend on four parameters $c_{1}, c_{2}, t_{0}, b$. However, in our discussion we will keep the parameters $\alpha$ and $\beta$ explicitly for both the M-theory and the string theory solutions.

\section{Direct dimensional reduction}

As mentioned in the introduction in order to correctly reproduce the string theory Sbranes we must start from a delocalized SM-brane solution and compactify the delocalized 
direction. So, instead of the form of the metric in (2.1), we start from the metric ansatz (this is exactly the same as in [9], with $q-k=1$ ),

$$
d s_{d}^{2}=-e^{2 \tilde{A}(t)} d t^{2}+e^{2 \tilde{B}(t)}\left(d x_{1}^{2}+\cdots+d x_{p+1}^{2}\right)+e^{2 \tilde{C}(t)} d \Sigma_{n-1, \sigma}^{2}+e^{2 \tilde{D}(t)} d y^{2}
$$

We take $\phi=0$. The equations of motion for gravity coupled to $n$-form field strength can be solved with the gauge condition,

$$
-\tilde{A}+(p+1) \tilde{B}+(n-1) \tilde{C}+\tilde{D}=0
$$

Using equations of motion and the gauge condition (3.2) the four functions $\tilde{A}, \tilde{B}, \tilde{C}$ and $\tilde{D}$ can be expressed as,

$$
\begin{aligned}
\tilde{A} & =(n-1) \tilde{g}(t)-\frac{p+1}{n-1} \tilde{f}(t)+n\left(\tilde{c}_{1} t+\tilde{c}_{2}\right) \\
\tilde{B} & =\tilde{f}(t) \\
\tilde{C} & =\tilde{g}(t)-\frac{p+1}{n-1} \tilde{f}(t)+\tilde{c}_{1} t+\tilde{c}_{2} \\
\tilde{D} & =-\frac{p+1}{n-1} \tilde{f}(t)+\tilde{c}_{1} t+\tilde{c}_{2}
\end{aligned}
$$

Note that the delocalized solution in [9] does not contain the linear part $\left(\tilde{c}_{1} t+\tilde{c}_{2}\right)$ in the above functions and so, will not give the correct string theory solution under direct dimensional reductions. The solution of the equations of motion give the functions $\tilde{f}(t)$ and $\tilde{g}(t)$ in the forms,

$$
\begin{aligned}
& \tilde{f}(t)= \frac{2}{\chi} \ln \frac{\tilde{\alpha}}{\cosh \left[\frac{\chi \tilde{\alpha}}{2}\left(t-t_{0}\right)\right]}+\frac{1}{\chi} \ln \frac{(d-2) \chi}{(n-1) b^{2}} \\
& \tilde{g}(t)=\left\{\begin{array}{lll}
\frac{1}{(n-2)} \ln \frac{\tilde{\beta}}{\sinh [(n-2) \tilde{\beta}|t|]}-\frac{n-1}{n-2}\left(\tilde{c}_{1} t+\tilde{c}_{2}\right), & \text { for } & \sigma=-1 \\
\pm \tilde{\beta} t-\frac{n-1}{n-2}\left(\tilde{c}_{1} t+\tilde{c}_{2}\right), & \text { for } & \sigma=0 \\
\frac{1}{(n-2)} \ln \frac{\tilde{\beta}}{\cosh [(n-2) \tilde{\beta} t]}-\frac{n-1}{n-2}\left(\tilde{c}_{1} t+\tilde{c}_{2}\right), & \text { for } & \sigma=+1
\end{array}\right.
\end{aligned}
$$

with the parameter relation given as follows,

$$
\frac{n-1}{n-2} \tilde{c}_{1}^{2}+\frac{(p+1)(d-2)}{n-1} \tilde{\alpha}^{2}-(n-1)(n-2) \tilde{\beta}^{2}=0
$$

Also, the form of the field strength is given as $F_{n}=b \epsilon\left(\Sigma_{n-1, \sigma}\right) \wedge d y$.

In $d=11$, eqs.(3.1) - (3.5) represent delocalized SM2-brane solution for $p=2, n=7$, $\chi=6$ and delocalized SM5-brane solution for $p=5, n=4, \chi=12$. The 11-dimensional solution can be dimensionally reduced to 10 -dimensions along $y$-direction by the following metric relation,

$$
\begin{aligned}
d s_{11}^{2} & =e^{-2 \phi / 3} d s_{10, s}^{2}+e^{4 \phi / 3} d y^{2} \\
& =e^{-\phi / 6} d s_{10}^{2}+e^{4 \phi / 3} d y^{2}
\end{aligned}
$$


Where the metric $d s_{10, s}^{2}$ in the first line is the string frame metric and $d s_{10}^{2}=e^{-\phi / 2} d s_{10, s}^{2}$ is the Einstein frame metric. By comparing (3.6) with (3.1) we obtain the dilaton as,

$$
\phi=\frac{3}{2}\left[-\frac{p+1}{n-1} \tilde{f}(t)+\tilde{c}_{1} t+\tilde{c}_{2}\right]
$$

This would be identified with the dilaton for the 10-dimensional solutions, namely, the localized SD2 and SNS5-brane solutions which we discuss separately below.

(a) $S M 2 \rightarrow S D 2$

Note that we have $p=2, n=7, a=0$ and $\chi=6$ for SM2-brane solution and $p=2$, $n=6, a=-1 / 2$ and $\chi=32 / 5$ for SD2-brane solution. So, from (3.7) we find,

$$
\phi=-\frac{1}{4} \ln \frac{\tilde{\alpha}}{\cosh \left[3 \tilde{\alpha}\left(t-t_{0}\right)\right]}-\frac{1}{8} \ln \frac{9}{b^{2}}+\frac{3}{2}\left(\tilde{c}_{1} t+\tilde{c}_{2}\right)
$$

for the reduced SM2-brane solution. On the other hand, we find from (2.1)

$$
\phi=-\frac{1}{4} \ln \frac{\alpha}{\cosh \left[\frac{16 \alpha}{5}\left(t-t_{0}\right)\right]}-\frac{1}{8} \ln \frac{256}{25 b^{2}}+\frac{15}{16}\left(c_{1} t+c_{2}\right)
$$

for the SD2-brane solution. So, identifying (3.8) with (3.9) we get,

$$
\tilde{\alpha}=\frac{16}{15} \alpha, \quad \tilde{c}_{1}=\frac{5}{8} c_{1}, \quad \tilde{c}_{2}=\frac{5}{8} c_{2}
$$

We also find $\tilde{\beta}=\beta$, by examining the function $\tilde{g}(t)$ (given in $(3.4)$ ) and $g(t)$ (given in (2.4)). With this identification the parameter relation (3.5) becomes,

$$
\frac{6}{5} \tilde{c}_{1}^{2}+\frac{9}{2} \tilde{\alpha}^{2}-30 \tilde{\beta}^{2}=0 \Rightarrow \frac{15}{32} c_{1}^{2}+\frac{128}{25} \alpha^{2}-30 \beta^{2}=0
$$

This is exactly the parameter relation (2.6) for the SD2-brane solution. The form of the 10-dimensional Einstein frame metric can be calculated from (3.6) as,

$$
d s_{10}^{2}=-e^{\phi / 6+2 \tilde{A}} d t^{2}+e^{\phi / 6+2 \tilde{B}}\left(d x_{1}^{2}+d x_{2}^{2}+d x_{3}^{2}\right)+e^{\phi / 6+2 \tilde{C}} d \Sigma_{6, \sigma}^{2}
$$

Now it can be easily checked using (3.8), (3.4) and (3.3) that

$$
\begin{aligned}
\frac{1}{6} \phi+2 \tilde{A} & =-\frac{9}{8} \tilde{f}+12 \tilde{g}+\frac{57}{4}\left(\tilde{c}_{1} t+\tilde{c}_{2}\right)=12 g-\frac{6}{5} f=2 A \\
\frac{1}{6} \phi+2 \tilde{B} & =\frac{15}{8} \tilde{f}+\frac{1}{4}\left(\tilde{c}_{1} t+\tilde{c}_{2}\right)=2 f=2 B \\
\frac{1}{6} \phi+2 \tilde{C} & =-\frac{9}{8} \tilde{f}+2 \tilde{g}+\frac{9}{4}\left(\tilde{c}_{1} t+\tilde{c}_{2}\right)=-\frac{6}{5} f+2 g=2 C
\end{aligned}
$$

Where in writing the last two expressions in the above we have used eqs.(3.4), (3.10), (2.4) and (2.3). So, the metric (3.12) takes exactly the same form as SD2-brane metric 
given in (2.1). The field strength also takes the same form as that of SD2-brane. We have thus shown how to obtain SD2-brane solution of type IIA string theory from a delocalized SM2-brane solution of M-theory under direct dimensional reduction.

(b) $S M 5 \rightarrow$ SNS5

Here we note that for SM5-brane solution $p=5, n=4, a=0$ and $\chi=12$ and for SNS5-brane solution $p=5, n=3, a=-1$ and $\chi=16$. So, from (3.7) we find that the dilaton takes the form,

$$
\phi=-\frac{1}{2} \ln \frac{\tilde{\alpha}}{\cosh \left[6 \tilde{\alpha}\left(t-t_{0}\right)\right]}-\frac{1}{4} \ln \frac{36}{b^{2}}+\frac{3}{2}\left(\tilde{c}_{1} t+\tilde{c}_{2}\right)
$$

for the reduced SM5-brane solution. On the other hand, we find from (2.1)

$$
\phi=-\frac{1}{2} \ln \frac{\alpha}{\cosh \left[8 \alpha\left(t-t_{0}\right)\right]}-\frac{1}{4} \ln \frac{64}{b^{2}}+\frac{3}{4}\left(c_{1} t+c_{2}\right)
$$

for the SNS5-brane solution. So, identifying (3.14) with (3.15) we get,

$$
\tilde{\alpha}=\frac{4}{3} \alpha, \quad \tilde{c}_{1}=\frac{1}{2} c_{1}, \quad \tilde{c}_{2}=\frac{1}{2} c_{2}
$$

Also, as before we have $\tilde{\beta}=\beta$. With this identification the parameter relation for the delocalized SM5-brane solution (3.5) takes the following form using (3.16),

$$
\frac{3}{2} \tilde{c}_{1}^{2}+18 \tilde{\alpha}^{2}-6 \tilde{\beta}^{2}=0 \Rightarrow \frac{3}{8} c_{1}^{2}+32 \alpha^{2}-6 \beta^{2}=0
$$

This is exactly the parameter relation of SNS5-brane solution given in (2.6). The 10dimensional Einstein frame metric can be obtained from (3.6) as,

$$
d s_{10}^{2}=-e^{\phi / 6+2 \tilde{A}} d t^{2}+e^{\phi / 6+2 \tilde{B}}\left(d x_{1}^{2}+\cdots+d x_{6}^{2}\right)+e^{\phi / 6+2 \tilde{C}} d \Sigma_{3, \sigma}^{2}
$$

It can be checked using (3.14), (3.4) and (3.3) that

$$
\begin{aligned}
\frac{1}{6} \phi+2 \tilde{A} & =-\frac{9}{2} \tilde{f}+6 \tilde{g}+\frac{33}{4}\left(\tilde{c}_{1} t+\tilde{c}_{2}\right)=6 g-6 f=2 A \\
\frac{1}{6} \phi+2 \tilde{B} & =\frac{3}{2} \tilde{f}+\frac{1}{4}\left(\tilde{c}_{1} t+\tilde{c}_{2}\right)=2 f=2 B \\
\frac{1}{6} \phi+2 \tilde{C} & =-\frac{9}{2} \tilde{f}+2 \tilde{g}+\frac{9}{4}\left(\tilde{c}_{1} t+\tilde{c}_{2}\right)=-6 f+2 g=2 C
\end{aligned}
$$

Where in writing the last two expressions in the above we have used eqs.(3.4), (3.16), (2.4) and (2.3). So, the metric (3.18) takes exactly the same form as SNS5-brane metric given in (2.1). The field strength also takes the same form as that of SNS5-brane solution. So, we have obtained SNS5-brane solution of type IIA string theory starting form the delocalized SM5-brane solution of M-theory by direct dimensional reduction. 


\section{Double dimensional reduction}

In the case of double dimensional reduction we compactify the M-theory along one of the space-like directions of the brane. But, it is clear that if we start from the isotropic M-brane solution given in (2.1), then the dilaton (3.6) in the reduced theory will not contain the linear time-dependent part (of the form $c_{1} t+c_{2}$ ) and we will not get the right form of the dilaton (2.1) of the string theory S-brane. However, we show that if we use the anisotropic (along the to be compactified direction) M-theory S-brane solution ${ }^{5}$, then we correctly reproduce the string theory S-brane under double dimensional reduction.

So, instead of (2.1), we take the metric ansatz of the M-theory S-brane solution as,

$$
d s_{d}^{2}=-e^{2 \hat{A}(t)} d t^{2}+e^{2 \hat{B}(t)}\left(d x_{1}^{2}+\cdots+d x_{p}^{2}\right)+e^{2 \hat{C}(t)} d \Sigma_{n, \sigma}^{2}+e^{2 \hat{D}(t)} d y^{2}
$$

where we identify $x_{p+1} \equiv y$ as the to be compactified direction. Here also, we take $\phi=0$. The equations of motion is the same as in eqs.(3) - (5) of ref. [9] with $\phi=a=0$. However, the non-vanishing components of the Ricci tensor for the above metric takes the form

$$
\begin{aligned}
R_{t t} & =-p\left(\ddot{\hat{B}}+\dot{\hat{B}}^{2}-\dot{\hat{A}} \dot{\hat{B}}\right)-n\left(\ddot{\hat{C}}+\dot{\hat{C}}^{2}-\dot{\hat{A}} \dot{\hat{C}}\right)-\left(\ddot{\hat{D}}+\dot{\hat{D}}^{2}-\dot{\hat{A}} \dot{\hat{D}}\right) \\
R_{x x} & =e^{2 \hat{B}-2 \hat{A}}\left[\ddot{\hat{B}}-\dot{\hat{A}} \dot{\hat{B}}+p \dot{\hat{B}}^{2}+n \dot{\hat{B}} \dot{\hat{C}}+\dot{\hat{B}} \dot{\hat{D}}\right] \\
R_{y y} & =e^{2 \hat{D}-2 \hat{A}}\left[\ddot{\hat{D}}-\dot{\hat{A}} \dot{\hat{D}}+p \dot{\hat{B}} \dot{\hat{D}}+n \dot{\hat{D}} \dot{\hat{C}}+\dot{\hat{D}}^{2}\right] \\
R_{a b} & =\left\{e^{2 \hat{C}-2 \hat{A}}\left[\ddot{\hat{C}}-\dot{\hat{A}} \dot{\hat{C}}+p \dot{\hat{B}} \dot{\hat{C}}+n \dot{\hat{C}}^{2}+\dot{\hat{C}} \dot{\hat{D}}\right]+\sigma(n-1)\right\} \bar{g}_{a b}
\end{aligned}
$$

where $\bar{g}_{a b}$ is the metric of the hyperspace $\Sigma_{n, \sigma}$ and the Ricci tensor for this space is given by, $\bar{R}_{a b}=\sigma(n-1) \bar{g}_{a b}$. The equations of motion simplifies under the gauge condition,

$$
-\hat{A}+p \hat{B}+n \hat{C}+\hat{D}=0
$$

Using this and the equations of motion, the above functions can be expressed as,

$$
\begin{aligned}
\hat{A} & =n \hat{g}(t)-\frac{p+1}{n-1} \hat{f}(t)+(n+1)\left(\hat{c}_{1} t+\hat{c}_{2}\right) \\
\hat{B} & =\hat{f}(t) \\
\hat{C} & =\hat{g}(t)-\frac{p+1}{n-1} \hat{f}(t)+\hat{c}_{1} t+\hat{c}_{2} \\
\hat{D} & =\hat{f}(t)+\hat{c}_{1} t+\hat{c}_{2}
\end{aligned}
$$

\footnotetext{
${ }^{5}$ This has also been recognized for $\sigma=-1$ in different supergravity S-brane solutions discussed in [10].
} 
By solving the equations of motion we obtain,

$$
\begin{aligned}
& \hat{f}(t)=\frac{2}{\chi} \ln \frac{\hat{\alpha}}{\cosh \left[\frac{\chi \hat{\alpha}}{2}\left(t-t_{0}\right)\right]}+\frac{1}{\chi} \ln \frac{(d-2) \chi}{(n-1) b^{2}}-\frac{1}{p+1}\left(\hat{c}_{1} t+\hat{c}_{2}\right) \\
& \hat{g}(t)= \begin{cases}\frac{1}{(n-1)} \ln \frac{\hat{\beta}}{\sinh [(n-1) \hat{\beta}|t|]}-\frac{n}{n-1}\left(\hat{c}_{1} t+\hat{c}_{2}\right), & \text { for } \sigma=-1 \\
\pm \hat{\beta} t-\frac{n}{n-1}\left(\hat{c}_{1} t+\hat{c}_{2}\right), & \text { for } \quad \sigma=0 \\
\frac{1}{(n-1)} \ln \frac{\hat{\beta}}{\cosh [(n-1) \hat{\beta} t]}-\frac{n}{n-1}\left(\hat{c}_{1} t+\hat{c}_{2}\right), & \text { for } \quad \sigma=+1\end{cases}
\end{aligned}
$$

where the parameters satisfy,

$$
\frac{p}{p+1} \hat{c}_{1}^{2}+\frac{(p+1)(d-2)}{n-1} \hat{\alpha}^{2}-n(n-1) \hat{\beta}^{2}=0
$$

The field strength is given as $F_{n}=b \epsilon\left(\Sigma_{n, \sigma}\right)$. In $d=11$, eqs.(4.1) - (4.6) represent the anisotropic SM2-brane solution for $p=2, n=7, \chi=6$ and anisotropic SM5-brane solution for $p=5, n=4, \chi=12$. The dimensional reduction is performed along the brane direction $y$ and the relation between the 11-dimensional metric and the 10-dimensional metric is as given in (3.6). By comparing (3.6) with (4.1) we obtain the dilaton as,

$$
\phi=\frac{3}{2} \hat{f}+\frac{3}{2}\left(\hat{c}_{1} t+\hat{c}_{2}\right)
$$

This would be identified with the dilaton for the 10-dimensional solution i.e. the localized SNS1 and SD4-brane solution and we discuss the two cases separately below.

(a) SM2 $\rightarrow$ SNS1

In this case we have $p=2, n=7, a=0, \chi=6$ for SM2-brane solution and $p=1$, $n=7, a=1, \chi=16 / 3$ for SNS1-brane solution (given in (2.1)). So, from (4.7) and (4.5) we obtain

$$
\phi=\frac{1}{2} \ln \frac{\hat{\alpha}}{\cosh \left[3 \hat{\alpha}\left(t-t_{0}\right)\right]}+\frac{1}{4} \ln \frac{9}{b^{2}}+\left(\hat{c}_{1} t+\hat{c}_{2}\right)
$$

for the dimensionally reduced anisotropic SM2-brane. On the other hand, from (2.1) we find,

$$
\phi=\frac{1}{2} \ln \frac{\alpha}{\cosh \left[\frac{8 \alpha}{3}\left(t-t_{0}\right)\right]}+\frac{1}{4} \ln \frac{64}{9 b^{2}}+\frac{3}{4}\left(c_{1} t+c_{2}\right)
$$

for the SNS1-brane. Identifying (4.8) with (4.9) we find,

$$
\hat{\alpha}=\frac{8}{9} \alpha, \quad \hat{c}_{1}=\frac{3}{4} c_{1}, \quad \hat{c}_{2}=\frac{3}{4} c_{2}
$$

and comparing the function $\hat{g}(t)$ in (4.5) and $g(t)$ in (2.1) we get $\hat{\beta}=\beta$. Now using (4.10) the parameter relation (4.6) reduces to,

$$
\frac{2}{3} \hat{c}_{1}^{2}+\frac{9}{2} \hat{\alpha}^{2}-42 \hat{\beta}^{2}=0 \Rightarrow \frac{3}{8} c_{1}^{2}+\frac{32}{9} \alpha^{2}-42 \beta^{2}=0
$$


This is exactly the parameter relation of SNS1-brane solution given in (2.6). The 10dimensional Einstein frame metric (3.6) takes the form,

$$
d s_{10}^{2}=-e^{\phi / 6+2 \hat{A}} d t^{2}+e^{\phi / 6+2 \hat{B}}\left(d x_{1}^{2}+d x_{2}^{2}\right)+e^{\phi / 6+2 \hat{C}} d \Sigma_{7, \sigma}^{2}
$$

Now using (4.7) and (4.4) we find,

$$
\begin{aligned}
\frac{1}{6} \phi+2 \hat{A} & =-\frac{3}{4} \hat{f}+14 \hat{g}+\frac{65}{4}\left(\hat{c}_{1} t+\hat{c}_{2}\right)=-\frac{2}{3} f+14 g=2 A \\
\frac{1}{6} \phi+2 \hat{B} & =\frac{9}{4} \hat{f}+\frac{1}{4}\left(\hat{c}_{1} t+\hat{c}_{2}\right)=2 f=2 B \\
\frac{1}{6} \phi+2 \hat{C} & =-\frac{3}{4} \hat{f}+2 \hat{g}+\frac{9}{4}\left(\hat{c}_{1} t+\hat{c}_{2}\right)=-\frac{2}{3} f+2 g=2 C
\end{aligned}
$$

Where in writing the last two expressions above we have used eqs.(4.5), (4.10), (2.4) and (2.3). We therefore find that the metric in (4.12) matches exactly with that of SNS1-brane solution given in (2.1). The 7-form field strength also matches trivially. This therefore shows that the double dimensional reduction of an anisotropic SM2-brane solution indeed correctly reproduces the SNS1-brane solution of type IIA string theory.

(b) $\mathrm{SM5} \rightarrow \mathrm{SD} 4$

Here also we employ the same procedure as in the previous subsection. In this case $p=5, n=4, a=0, \chi=12$ for SM2-brane solution and $p=4, n=4, a=1 / 2$, $\chi=32 / 3$ for SD4-brane solution (given in (2.1)). The dilaton for the dimensionally reduced anisotropic SM5-brane can be obtained from (4.7) and (4.5) as,

$$
\phi=\frac{1}{4} \ln \frac{\hat{\alpha}}{\cosh \left[6 \hat{\alpha}\left(t-t_{0}\right)\right]}+\frac{1}{8} \ln \frac{36}{b^{2}}+\frac{5}{4}\left(\hat{c}_{1} t+\hat{c}_{2}\right)
$$

The form of the dilaton for the SD4-brane solution can be obtained from (2.1) as,

$$
\phi=\frac{1}{4} \ln \frac{\alpha}{\cosh \left[\frac{16 \alpha}{3}\left(t-t_{0}\right)\right]}+\frac{1}{8} \ln \frac{256}{9 b^{2}}+\frac{15}{16}\left(c_{1} t+c_{2}\right)
$$

Identifying these two we obtain,

$$
\hat{\alpha}=\frac{8}{9} \alpha, \quad \hat{c}_{1}=\frac{3}{4} c_{1}, \quad \hat{c}_{2}=\frac{3}{4} c_{2}
$$

Also as before we get $\hat{\beta}=\beta$. So, using (4.16) the parameter relation for SM5-brane solution (4.6) gives,

$$
\frac{5}{6} \hat{c}_{1}^{2}+18 \hat{\alpha}^{2}-12 \hat{\beta}^{2}=0 \Rightarrow \frac{15}{32} c_{1}^{2}+\frac{128}{9} \alpha^{2}-12 \beta^{2}=0
$$


We note that this is precisely the parameter relation for the SD4-brane solution as can be seen from (2.6). Now we can check whether the dilaton as well as the parameter identification correctly reproduces the 10-dimensional SD4-brane metric from (3.6). The Einstein frame metric in (3.6) has the form,

$$
d s_{10}^{2}=-e^{\phi / 6+2 \hat{A}} d t^{2}+e^{\phi / 6+2 \hat{B}}\left(d x_{1}^{2}+\cdots+d x_{5}^{2}\right)+e^{\phi / 6+2 \hat{C}} d \Sigma_{4, \sigma}^{2}
$$

It can be easily checked using (4.7) and (4.16) that

$$
\begin{aligned}
& \frac{1}{6} \phi+2 \hat{A}=-\frac{15}{4} \hat{f}+8 \hat{g}+\frac{41}{4}\left(\hat{c}_{1} t+\hat{c}_{2}\right)=-\frac{10}{3} f+8 g=2 A \\
& \frac{1}{6} \phi+2 \hat{B}=\frac{9}{4} \hat{f}+\frac{1}{4}\left(\hat{c}_{1} t+\hat{c}_{2}\right)=2 f=2 B \\
& \frac{1}{6} \phi+2 \hat{C}=-\frac{15}{4} \hat{f}+2 \hat{g}+\frac{9}{4}\left(\hat{c}_{1} t+\hat{c}_{2}\right)=-\frac{10}{3} f+2 g=2 C
\end{aligned}
$$

In the last two expressions above we have used eqs.(4.5), (4.16), (2.4) and (2.3). This shows that we indeed obtain SD4 metric, dilaton, and the 4-form field strength (this matches trivially) starting from the anisotropic SM5-brane solution by double dimensional reduction.

\section{Conclusion}

In this paper we have studied the direct as well as the double dimensional reduction of the M-theory S-branes to string theory S-branes. This procedure is well-known for the usual time-like branes, but we pointed out that the reduction procedure is quite different for the S-branes. The difference can be understood since both M-theory and string theory S-brane solutions are known explicitly and it is also known that M-theory solutions are characterized by two parameters whereas the string theory solutions are characterized by four parameters. Although the physical meaning of these parameters is not well understood, it is clear that new parameters can not be produced by only dimensional reduction. New parameters can be introduced if instead of localized SMbrane solutions given in eq.(2.1), we start from delocalized (in one of the transverse to be compactified space-like directions of the brane) or anisotropic (in one of the longitudinal to be compactified directions of the brane) solutions. Inspection of the equations of motion suggests that the delocalization or the anisotropization (along one direction which is to be compactified) of the SM-branes produces exactly the required number of parameters for the string theory S-branes. This is exactly what we have done and thus we have shown that these solutions i.e. SM2 and SM5 correctly reproduces the string theory Sbranes namely, SD2 and SNS5-brane under direct dimensional reduction and SNS1 and 
SD4-brane under double dimensional reduction. For direct dimensional reduction we have used the delocalized solution and for double dimensional reduction we have used the anisotropic solution.

It should be noted that since the dilaton of the string theory is related to the radius of the compactified eleventh dimension by $e^{2 \phi / 3} \sim R_{11}$ and it is time dependent, it is quite

crucial to see whether the compactification is achieved by looking at whether $e^{\phi} \sim R_{11}^{3 / 2} \ll$ 1. As $t$ varies, we notice from (3.7) and (4.7) that it would depend on the parameters $\alpha$ and $c_{1}$. It is conceivable that there might exist some range of $t$, where $R_{11}$ remains large and we will see the full 11-dimensional theory instead of the 10-dimensional one. However, in 11 dimensions the decompactified (or 'uplifted') string theory S-branes would not be the localized, isotropic SM-branes, but the delocalized or anisotropic branes.

\section{Acknowledgements}

I would like to thank Somdatta Bhattacharya and Sudipta Mukherji for discussions.

\section{References}

[1] M. Gutperle and A. Strominger, "Space-like branes", JHEP 04 (2002) 018, hep-th/0202221.

[2] A. Sen, "Non-BPS states and branes in string theory", hep-th/9904207.

[3] A. Strominger, "The dS/CFT correspondence", JHEP 10 (2001) 034, hep-th/0106113.

[4] V. Balasubramanian, J. de Boer and D. Minic, "Mass, entropy and holography in asymptotically de Sitter space", hepth/0110108.

[5] M. Park, "Statistical entropy of three dimensional Kerr-de Sitter space", Phys. Lett. B440 (1998) 275, hep-th/9806119; "Symmetry algebras in Chern-Simons theories with boundary: canonical approach", Nucl. Phys. B544 (1999) 377, hep-th/9811033.

[6] J. Khoury, B. Ovrut, N. Seiberg, P. Steinhardt and N. Turok, "From big crunch to big bang", Phys. Rev. D65 (2002) 086007, hep-th/0108187; V. Balasubramanian, F. Hassan, E. Keski-Vakkuri and A. Naqvi, "A Space-time orbifold: a toy model for cosmological singularity", hep-th/0202187; N. Nekrasov, "Milne universe, tachyons 
and quantum group", hep-th/0203112, L. Cornalba and M. Costa, "A new cosmological scenario in string theory", Phys. Rev. D66 (2002) 066001, hep-th/0203031; L. Cornalba, M. Costa and C. Kounnas, "A resolution of the cosmological singularity with orientifolds", Nucl. Phys. B637 (2002) 378, hep-th/0204261. O. Aharony, M. Fabinger, G. Horowitz and E. Silverstein, "Clean time dependent string backgrounds from bubble baths", hep-th/0204158; H. Liu, G. Moore and N. Seiberg, "Strings in a time dependent orbifold", hepth/0204168.

[7] H. Lu, S. Mukherji, C. Pope and K. Xu, "Cosmological solutions in string theories", Phys. Rev. D55 (1997) 7926, hep-th/9610107; H. Lu, S. Mukherji and C. Pope, "From p-branes to cosmology", Int. J. Mod. Phys. A14 (1999) 4121, hep-th/9612224; A. Lukas, B. Ovrut and D. Waldram, "Cosmological solutions of type II string theory", Phys. Lett. B393 (1997) 65, hep-th/9608195; A. Lukas, B. Ovrut and D. Waldram, "String and M-theory cosmological solutions with Ramond forms", Nucl. Phys. B495 (1997) 365, hep-th/9610238; K. Behrndt and S. Forste, "String KaluzaKlein cosmology", Nucl. Phys. B430 (1994) 441, hep-th/9403179.

[8] P. Townsend and M. Wohlfarth, "Accelerating cosmologies from compactification", hep-th/0303097; N. Ohta, "Accelerating cosmologies from S-branes", hep-th/0303238; S. Roy, "Accelerating cosmologies from M/string theory compactifications", hep-th/0304084; M. Wohlfarth, "Accelerating cosmologies and a phase transition in M-theory", hep-th/0304089, R. Emparan and J. Garriga, "A note on accelerating cosmologies from compactifications and S-branes", hep-th/0304124; C. Chen, P. Ho, I. Neupane and J. Wang, "A note on acceleration from product space compactification", hep-th/0304177, M. Gutperle, R. Kallosh and A. Linde, "M/String theory, S-branes and accelerating universe", hep-th/0304225 M. Ito, "On the solutions to accelerating cosmologies", hep-th/0305130.

[9] C. -M. Chen, D. Gal'tsov and M. Gutperle, "S-brane solutions in supergravity theories", Phys. Rev. D66 (2002) 024043, hep-th/0204071.

[10] M. Kruczenski, R. Myers and A. Peet, "Supergravity S-branes", JHEP 05 (2002) 039, hep-th/0204144.

[11] S. Roy, "On supergravity solutions of space-like Dp-branes", JHEP 08 (2002) 025, hep-th/0205198.

[12] N. Deger and A. Kaya, "Intersecting S-brane solutions of D = 11 supergravity", JHEP 07 (2002), hep-th/0206057; J. Wang, "Spacelike and time dependent branes from 
DBI", JHEP 10 (2002) 037, hep-th/0207089; F. Quevedo, S. Rey, G. Tasinato and I Zavala, "Cosmological spacetimes from negative tension brane backgrounds", JHEP 10 (2002) 028, hep-th/0207104; A. Buchel, P. Langfelder, and J. Walcher, "Does the tachyon matter?", Annals Phys. 302 (2002) 78, hep-th/0207235; V. Ivashchuk, "Composite S-brane solutions related to Toda type system", Class. Quant. Grav. 20 (2003) 261, hep-th/0208101; A. Strominger, "Open string creation by S-branes", hep-th/0209090 F. Quevedo, G. Tasinato and I. Zavala, "S-branes, negative tension branes and cosmology, hep-th/0211031; K. Hashimoto, P. Ho and J. Wang, "S-brane actions", hep-th/0211090 A. Buchel and J. Walcher, "The tachyon does matter", hep-th/ 0212150; N. Ohta, "Intersection rules for S-branes", hep-th/0301095 C. Burgess, P. Martineau, F. Quevedo, G. Tasinato and I. Zavala, "Instabilities and particle production in S-brane geometries", hep-th/0301095 A. Maloney, A. Strominger and X. Yin, "S-brane thermodynamics", hep-th/0302146, F. Leblond and A. Peet, "SD-brane gravity fields and rolling tachyons", hep-th/0303035; K. Hashimoto, P. Ho, S. Nagaoka and J. Wang, "Time evolution via S-branes", hep-th/0303172 N. Deger, "Non-standard intersections of S-branes in $D=11$ supergravity", hep-th/0303232 A. Buchel and J. Walcher, "Comments on supergravity description of S-branes", hep-th/0305055, F. Leblond and A. Peet, "A note on the singularity theorem for supergravity SD-branes", hep-th/0505059.

[13] H. Lu, C. Pope and K. Stelle, "Vertical versus diagonal dimensional reductions for p-branes", Nucl. Phys. B481 (1996) 313.

[14] M. Duff, P. Howe, T. Inami and K. Stelle, "Superstrings in D=10 from supermembranes in D=11", Phys. Lett. B191 (1987) 70.

[15] N. Ohta, "A study of accelerating cosmologies from superstring/M theories", hep-th/0304172. 